\title{
GENERATIVE MODELING, VIRTUAL REALITY AND HBIM INTERACTION: IMMERSIVE ENVIRONMENT FOR BUILT HERITAGE: CASE STUDY OF SHAIKH ISA BIN ALI HOUSE, BAHRAIN
}

\author{
F. Banfi ${ }^{1 *}$, R. Brumana ${ }^{1}$, A. Aljishi ${ }^{2}$, N. Al Sayeh ${ }^{2}$, M. Santana Quintero ${ }^{3}$, B. Cuca ${ }^{1}$, D. Oreni ${ }^{1}$, C. Midali ${ }^{4}$ \\ ${ }^{1}$ Dept. of Architecture, Build Environment and Construction Engineering, Politecnico di Milano, Milan, Italy \\ (fabrizio.banfi ; raffaella.brumana)@polimi.it \\ ${ }^{2}$ Bahrain Authority of Culture and Antiquities, P.O box 2199, Manama, Kingdom of Bahrain, ahmed.aljishi@culture.gov.bh , \\ noura.sayeh@culture.gov.bh \\ ${ }^{3}$ Carleton Immersive Media Studio (CIMS), 1125 Colonel by Drive, Ottawa, On, K1S 5B6 Canada, mario.santana@carleton.ca \\ ${ }^{4}$ Politecnico di Milano, Piazza Leonardo da Vinci 32, Milan, Italy, chiara.midali@polimi.it
}

Commission WG II/8, WG III/5, WG V/1

KEY WORDS: Scan-to-BIM, Generative modeling, Heritage Building Information Modeling (HBIM), Grade of Generation (GOG), Bioclimatic Architecture, Virtual Reality (VR), Interactivity.

\begin{abstract}
:
The innovation in Digital Cultural Heritage (DCH) shows an emergence of innovative methods and initiatives for the generation of complex historical models to which are linked useful information for specialists of architecture, construction and engineering, but also user community interested in cultural heritage. This paper presents an improvement in Historic Building Information Modeling (HBIM) turned into models for mobile apps based on virtual reality (VR) addressed to enhance the communication of Architectural Heritage. The opportunity of a collaboration with the Bahrain Authority for Culture and Antiquities (BACA) experts under the coordination of the Carleton University ${ }^{1}$ in a training project aimed at improving documentation skills for conservation and preventive maintenance of built heritage provided the availability of an extraordinary richness of data and surveying on the Shaikh Isa Bin Ali House. The case study of the Shaikh Isa Bin Ali House is an outstanding example of Bahrain architecture and one of the oldest surviving building in Muharraq, listed under the UNESCO sites ${ }^{2}$, now transformed in a Museum, where the visit does not allow to perceive the richness of the traditions of the constructions, beside the beautiful architecture. The building was the seat of the ruler of Bahrain from 1869 until his death in 1932 and represented an interesting response to local building materials and climate. This vernacular architecture solved not only the climate problems but combined the solution with beauty, physical and social functionality. This research carries out digital preservation through HBIM and switches to Cloud and VR platforms to allow users to enter a 3D immersive scene, using models as a vehicle of content information.
\end{abstract}

\section{INTRODUCTION}

The house of Shaykh Isa bin Ali Al-Khalifa is located in Muharraq; it is considered to be the oldest in Bahrain. It was built ca. 1840 (Yarwood, 2005) as a private residence for the grandson of Shaykh Ahmed Ali Fatih (reg. 1783-1794), conqueror of Bahrain. In 1869, under Shaykh Isa bin Ali Khalifa (reg. 1869-1932), it was used as both the ruler's residence and the centre of government. The house was then inhabited by his son, Abdallah, until his death in 1972. Between 1976 and 2002 it underwent several modern interventions.

The building occupies a complete city block, and it has a rectangular shape. Its exterior walls are up to one meter thick constructed of mud bricks covered with gypsum, moderating the extreme climatic conditions. The house is divided into four quarters, each of which arranged around a courtyard.

The building has two entrances, the family entrance to the north and the guest entrance to the east. The majority of the rooms occupy the ground floor, while the summer rooms are found on the first-floor terrace. The house is characterized by delicate arches, intricate wooden doors and palm-trunk ceilings. The summer breezes are caught on the roof to provide air movement over the surface of the skin in the worst conditions of the heat and humidity, for this reason, most important pavilions for living and reception have a counterpart on the first floor. Ingenious and varied are often the methods by which cool comfort is obtained in this hot, humid climate (Nasssar et al. 2017). The building is fascinating. Not merely do it represent an apparent response to local building materials and climate, but it has a unique architectural character: clear, logical statement of the structure in a system which is articulated and proportioned according to the principles of classical Islamic architecture a thousand years old (Fig. 1).

In the early seventies Shaikha Haya Al-Khalifa, Director of Archaeology and Museums, saved the building because it meant

\footnotetext{
1 Project Title: Documentation of Built Heritage for the Bahrein Authority for Culture and Antiquities (BACA), Principal Investigator Prof. Mario Quintero Santana; Co-Investigator (collaborating) Prof. Luigi Barazzetti;

${ }^{2}$ UNESCO site: Shaikh Isa Bin Ali House is part of a larger programme of valorization of the heritage buildings, testifying the typical house constructions in Bahrein.
}

*Corresponding author 
to be demolished and transformed into a public square (General Conservation Directives, 2017).

The disfiguration of all later palaces makes Shaykh Isa bin Ali Al-Khalifa a unique witness of its time, it deserves to be investigated in depth with the help of new technologies.

In recent years, interest in smart technologies has increased; mobile devices have an important role in appealing the interaction between people and DCH (Santana et al., 2014) (Ioannides et al., 2017). The use of interactive technologies such as VR has changed that perception, and it opened up these spaces to a new audience. It has been used to construct virtual walkthroughs of these sites which intensify the visitor's experience. This new tool is a useful way to encourage more people to visit heritage sites. Because Building Information Modeling (BIM) is immediately available in $3 \mathrm{D}$, there is a direct connection between the digital model and advanced visualisation techniques based on VR.
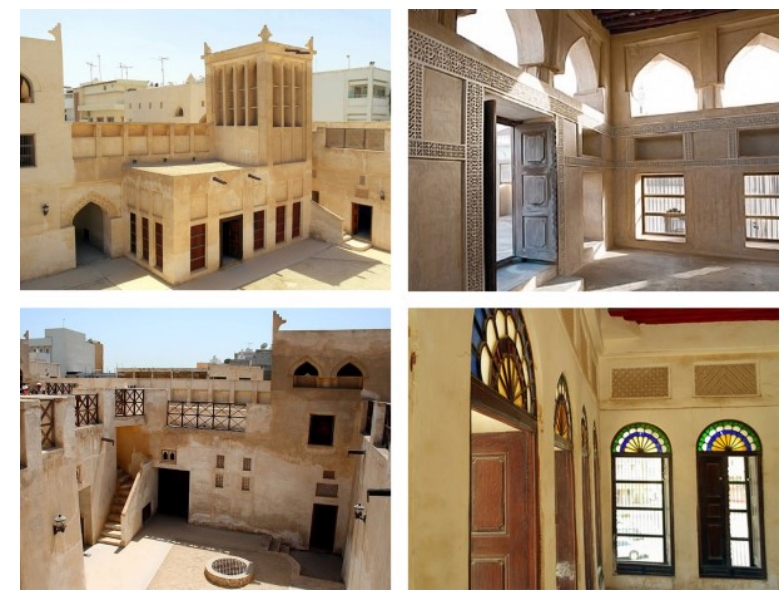

Figure 1. The house of Shaykh Isa bin Ali Al-Khalifa and its unique architectural elements.

\section{RELATED WORKS AND RESEARCH OBJECTIVES}

The Bahrain Pearling Pathway is a serial cultural heritage site inscribed on the UNESCO World Heritage List in 2012 (Meskell, 2013). It consists of three oyster beds in the Bahrain northern waters, a segment of the coast and the seafront $\mathrm{Bu}$ Mahir fortress in the southern tip of Muharraq Island, and 17 buildings in Muharraq historical town connected by a $3.5 \mathrm{~km}$ visitor pathway. The buildings listed were the residences and majlises of rich pearl merchants, along with shopping establishments, storage houses and the Siyadi family mosque. Among these properties, there is Shaikh Isa Bin Ali House.

The documentation project has been developed within this framework to support the Conservation and Urban Economic Revival Project in the city of Muharraq. The digital documentation of the house has been required to develop informative conservation and maintenance actions but also the decorative apparatus, spatial design and materials employed as well as to the innovative building solution adopted to adapt to the local hot climate. The project included several conservation stages. Currently, the Bahrain Authority for Culture and Antiquities (BACA) is taking care of its conservation and maintenance.
2.1 The availability of surveying and restitutions coming from the cooperation programme

The surveying of the House in the framework of the collaboration training project with BACA experts, aimed at improving documentation skills for conservation and preventive maintenance of built heritage. The surveying has been carried out by a multidisciplinary team of experts from (Carleton University, Canada) and the DABC-Gicarus Lab (Politecnico di Milano, Italy), employing digital techniques, aerial and terrestrial photogrammetry, 3D orthophotos, LIDAR data acquisition integrated on a geodetic network (Barazzetti et al., 2017): it has been produced an extraordinary richness of available data, collecting the geometric information and details: plans, cross sections, elevations, and orthophotos at the scale 1:50, but with an accuracy of the scale 1:20. A set of technical drawings was produced for each building; the main house and the Coffee House. The produced deliverables consist of two plans, six cross sections and four external elevations at the scale 1:50. An orthophoto of the roof at the scale 1:100, orthophotos of all the rooms at the scale 1:20 and four orthophotos of the external facades at the scale 1:50. The main building occupies a complete city block, an area of $1700 \mathrm{~m}^{2}$. The majority of the rooms (36 indoor spaces) occupy the ground floor, while the summer rooms (9 indoor spaces) are found on the first-floor terrace. The total volume is of almost $6130 \mathrm{~m}^{3}$. The restitution of the surveying and the drawing output have been addressed to highlight the different thickness of the walls, geometrical anomalies, different alignments among the different building elements, different proportions, construction details, taking in account all the richness coming from the TLS cloud scans without simplifying and rectifying the geometry obtained from the data acquisition (Oreni et al., 2013).
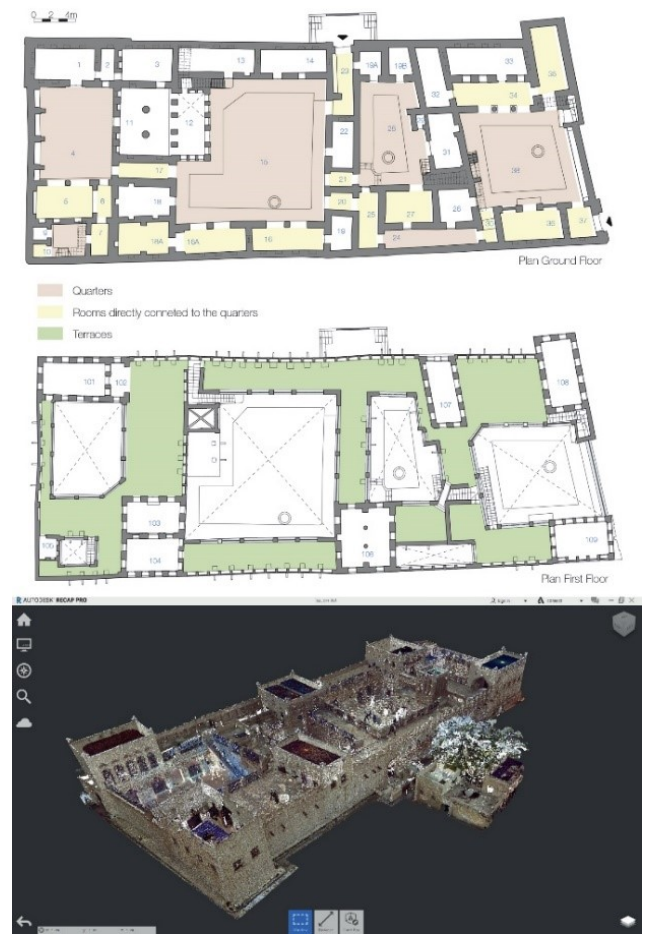

Figure 2. The point cloud project of The house of Shaykh Isa bin Ali Al-Khalifa and the as-found drawings produced during the process. 
Several digital techniques, including aerial (drone) and terrestrial photogrammetry, rectifying photography, total station and laser scanning have been used for documenting the building. Figure 2 shows the first phase (post-processing) applied to improve primary data sources (point clouds data and geodetic network) and secondary data sources (2D CAD drawings, geometric analysis, historical records) for the HBIM generation.

\subsection{From the surveying to HBIM modelling}

Thanks to the subdivision of the point clouds into layers (slices), it has been possible to improve the identification of each element. The cleaning process of point clouds required the integrated use of Autodesk Autocad, Recap and Mc Nell Rhinoceros for the production of plans, elevations and cross sections. Thanks to the application of the data processing based on the integration of the generative modeling and BIM software has been possible to obtain an accurate and usable HBIM model able to embody the complexity of the geometry and the given accuracy $(2-4 \mathrm{~mm})$.

The modeling of the architectural and structural elements, unique in their kind, required the application of different grades of generation (GOG) (Banfi 2017) with the aim of generating a model capable of transforming point clouds into parametric objects (Scan-to-BIM process). The semantic enrichment of each object has been enabled thanks to the application of different modeling requirements able to integrate and connect different types of information at different levels of detail (LOD) (Brumana et al., 2018a). Furthermore, each object has also been checked qualitatively. Thanks to the development of an automatic verification system (AVS) (Banfi et al., 2018) it was possible to calculate the grade of accuracy (GOA) of every single element, reaching a value of the standard deviation of about $1 \mathrm{~mm}$ respect to the surveyed data. This verification allowed to share the reliability of the model for every single area of the building. In particular, an interactive VR project has been developed to facilitate the understanding of the heritage of the cultural assets by the citizens, tourists and non-experts. The current Bahrain Authority for Culture and Antiquities (BACA) intends to insert four areas of exhibition spaces turning this monument in a sort of "House Museum". Those spaces are selected so that they would exhibit various aspects of the Shaikh Isa Bin Ali and his family. Movable antiques that belong to the ruling period, as well as interpretative items, are being acauired and nrenared for set such exhibition.

Technological devices can give a precious contribution helping to establish an appropriate link among the conservation process and purposes and its communication aiming to create a circular chain to improve awareness raising of the multiplicity of the evidence highlighted by the HMIM and a value-centred conservation of the authenticity of the materials, the construction techniques characterizing all the construction phases of the House, together with their functions. The provides dynamic ways to visual storytelling. For these reasons, this research supports the initiative by proposing technological measures to improve the overall effectiveness and the dissemination process.

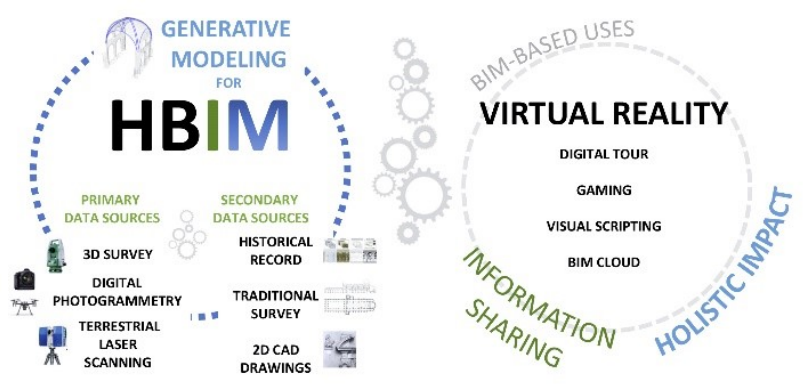

Figure 3. The research objective and the generative workflow applied to the research case study.

\section{RESEARCH METHODOLOGY}

3.1 Scan-to BIM process to entail heritage complexity: novel grades of generation (GOG9 and 10) and accuracy (GOA)

This study summarises the generative processes through the use of specific Grades of Generation (GOG) (Banfi 2017) oriented to improve the use of HBIM for different disciplines and purposes, with an exact correspondence with the building's 3D survey. In particular, using GOG 9 and GOG 10 , it is possible to create irregular, and complex objects from dens point clouds (3D scans). In this heritage building, all the walls have a marked irregular shape, so GOGs certainly come in useful.

The slice extraction procedure (GOG 9) from the point cloud has led to a skeleton of the building (3D wireframe). Once completed this slicing process it has been possible to interpolate the 3D edges with the scan's points (GOG 19) obtaining a NURBS model with a Grade of Accuracy (GOA) of $1 / 2 \mathrm{~mm}$.

Thanks to the GOGs flexibility, each irregular BIM object (wall, floor, roof) has been created with an integrated approach based on four modeling steps able to represent the building's complexity accurately:

- the first step was based on the integration of multiple GOGs (from 1 to 7) and the use of GOG 8 for the generation of empty spaces, openings and irregular wall portions in order to realise subtractions where were needed;

- the second step consists of the integrated use of GOG 9 and 10 in order to improve the GOA of irregular and curved shapes.

- the third step allowed to develop an HBIM model enriched with information and analysis such as the bioclimatic behaviour of the ventilation system, where all the "ventilation structure" and bioclimatic elements have been translated into BIM objects.

- the fourth step, thanks to the integration of more specific features such as material analyses, stratigraphy, physical and thermal characteristics (information mapping), allowed the transformation of HBIM model into an information system. Thanks to access to a cloud platform, schedules, and computing automatically obtained from the HBIM model (data extraction) has been shared to different users, thus improving information sharing during the process.

The second step required different modeling tests able to establish a sustainable generative process open to different 
BIM-based uses. As we well know, the modeling software is mainly divided into two large families:

- Generative modeling software is based on free-form modeling. The most commonly used solutions are McNeel Rhinoceros, Autodesk 3DS Max, Blender, Autodesk Maya, SketchUp Pro and Autodesk Autocad;

- $\quad$ BIM software is based on 3D objects corresponding to the building components. The most commonly used solutions are Autodesk Revit, Graphisoft Archicad and AECOsim Building Designer.

In light of this, in order to bridge the modeling gap of BIM software (not oriented to generate complex heritage buildings), the key idea of this research was to integrate the NURBS modeling in the HBIM process. In particular, the first eight GOGs were applied for to not complex elements (Step 1).

Unlike the first seven GOGs (extrusion, edit profile, edit profile with empty spaces, sweep, reveal, extrusion of irregular profile and revolve), GOG 8 has allowed subtraction procedures complex volumetric portions from HBIM objects such as historical loggias, openings, wall deformations with the aim of increasing the GOA of each element.

This technique turned out useful because it allowed optimising the generation of complex elements avoiding to use other modeling software and exchange formats such as acis or step format. Figure 4 shows all the elements created by the first eight GOGs, underlining the importance of how high levels of complexity can be achieved using a single application (in this case Autodesk Revit).

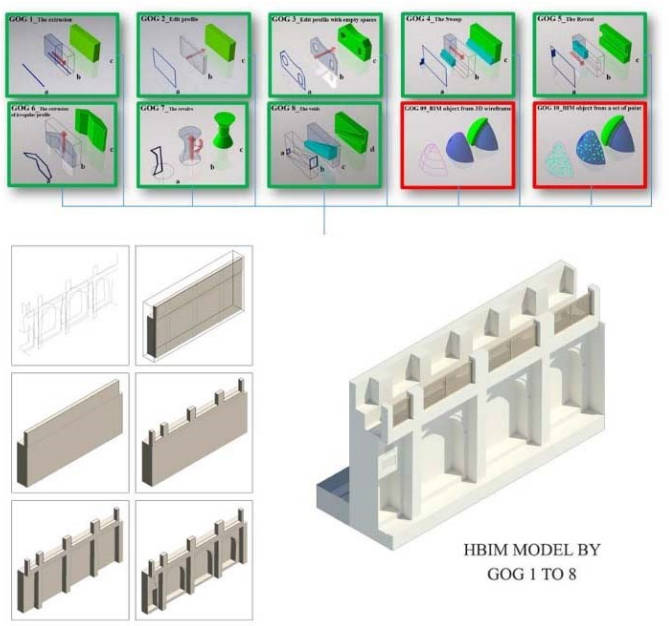

Figure 4. The first step based on the first eight GOGs (From the simple extrusion to the volumetric subtraction) allows creating complex heritage elements within Autodesk Revit.

The second step required the use of novel Scan-to-BIM modeling requirements. GOG 9 and GOG 10 have shown great flexibility for transforming point cloud data into BIM objects during the last few years (Brumana et al. 2018 b) (Stanga et al. 2017).

In particular, GOG 9 has been applied for the extraction of geometric primitives corresponding to the physical boundaries of every single element. This technique leads to the creation of three-dimensional wireframe models.

The identification of the physical boundaries of each element corresponds to the mathematical 3D edges required by the NURBS algorithms for the generation of complex 3D objects.

The main difference between the GOG 9 and the GOG 10 lies in the use of specific geometric primitives such as lines, splines and points.

On one side the GOG 9 requires the definition of geometric primitives corresponding to the external and internal edges of every single element, on the other side the GOG 10 allows to considerably reduce the costs and times of modeling thanks to the interpolation of the 3D scan's points for the automatic generation of complex NURBS models.

As evidenced by different studies and applications in the field of prototyping, industrial design, 3D animation, reverse engineering, NURBS modelig allows the creation of any shape. Reverse engineering processes lay its foundation for the use of point clouds to improve industrial products. The reverse process (from point clouds to $3 \mathrm{D}$ object) was the key for the development of GOG 10, oriented to generate historical elements and heritage buildings from 3D scans. As a result, complex and curved structural elements such as vaults, irregular walls, historical decorations have been realised thanks to the application of GOG 10. Figure 5 shows the main difference between GOG 9 and 10 and how a point cloud can be transformed into a parametric object bridging the modeling gaps within BIM software. In this case, the second step required the use of MC Neel Rhinoceros (generative modeling) and Autodesk Revit (HBIM parametrisation).
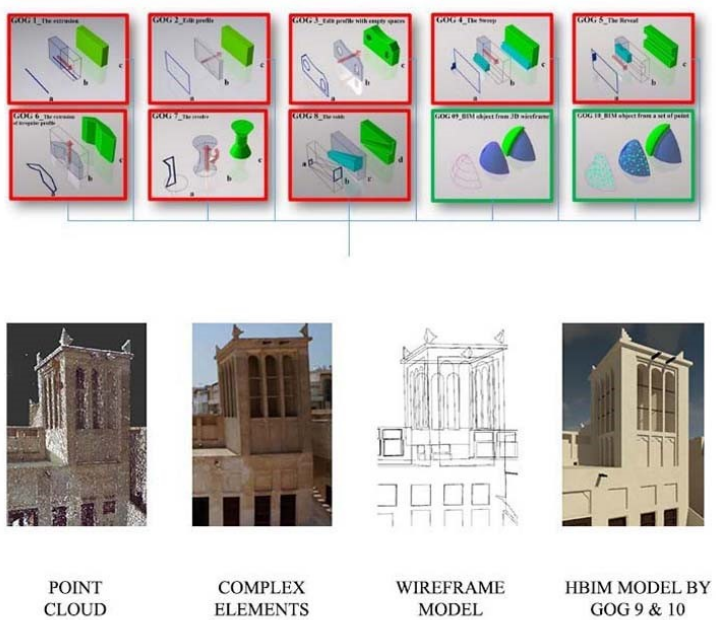

$$
\begin{aligned}
& \text { POINT } \\
& \text { CLOUD }
\end{aligned}
$$
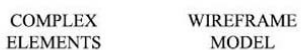

HBIM MODEL BY ELEMENTS GOG 9 \& 10

Figure 5. The second step based on GOG 9 and GOG 10 allows the generation of complex heritage elements using NURBS algorithms and BIM software.

The third step was the semantic model enrichment with different types of information. The generative process based on the creation of NURBS models required a processing stage based on the transformation of a geometric model into a parametric model. This step, in technical jargon, is called 'Parametrization'. In particular, it was possible to convert complex NURBS models into Autodesk Revit automatically by 
defining new interoperable schemas based on different exchange formats such as acis, step and dwg format.

In recent years, this parameterisation process of Scan-to-BIM objects has produced some interesting results in different disciplinary fields such as finite element analysis (Barazzetti et al., 2015) CoSIM (Trani et al.), infrastructure and monitoring (Martinelli et al., 2018).

Step 3 lays its foundation on the alternative method of importing the NURBS model into Autodesk Revit.

The limited set of modeling tools in Autodesk Revit is oriented to create and manage architectural and structural elements for new buildings. For the most part, the Revit modeling tools are difficult to use and do not allow the transformation of point clouds into BIM objects. Thanks to the establishment of GOG 9 and GOG 10 has been possible to bridge the generative modeling gap in Autodesk Revit. Once we have obtained 'unique' HBIM objects from point clouds with a proper GOA (Standard deviation 1,2 $\mathrm{mm}$ ) has been possible to improve the information mapping for each type of architectural component.

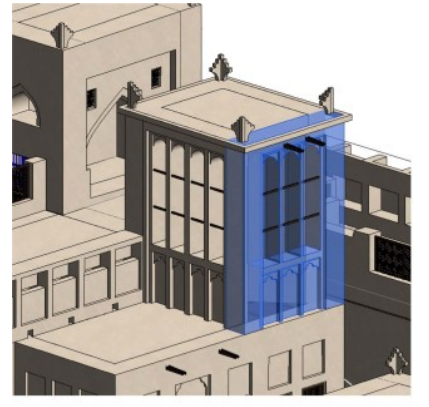

Example information linked to a wal

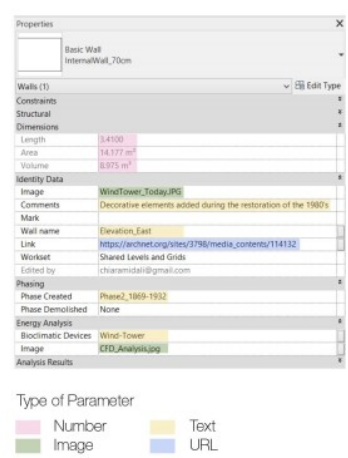

Figure 6. The third step is based on HBIM enrichment. The information mapping allows improving the different type of BIM-based analysis such as material stratigraphy, physical and thermal analyses.

\subsection{HBIM oriented to highlighting the Bioclimatic architecture}

The different bioclimatic architectural elements and solutions (wind tower, orientation, different distributions across the year in the use of the rooms, have been generated obtaining a Heritage Information Model of the richness 'bioclimatic culture': all the 'ventilation structures' and bioclimatic elements have been traduced in objects (walls, the typical opening and wooden windows, covering systems, together with the decorative apparatus). An HBIM representation of the bioclimatic behaviour of the ventilation system has been developed.

The level of information (LOI) has used to indicate the grade of the data that is linked to the BIM family: the integration of more specific features (such as material analyses, stratigraphy, physical and thermal characteristics) and the proper generation of 'unique' HBIM objects by GOG 9 and 10 have allowed the bi-directionally link between 3D object and new type of data, ensuring a precise automatic computing.
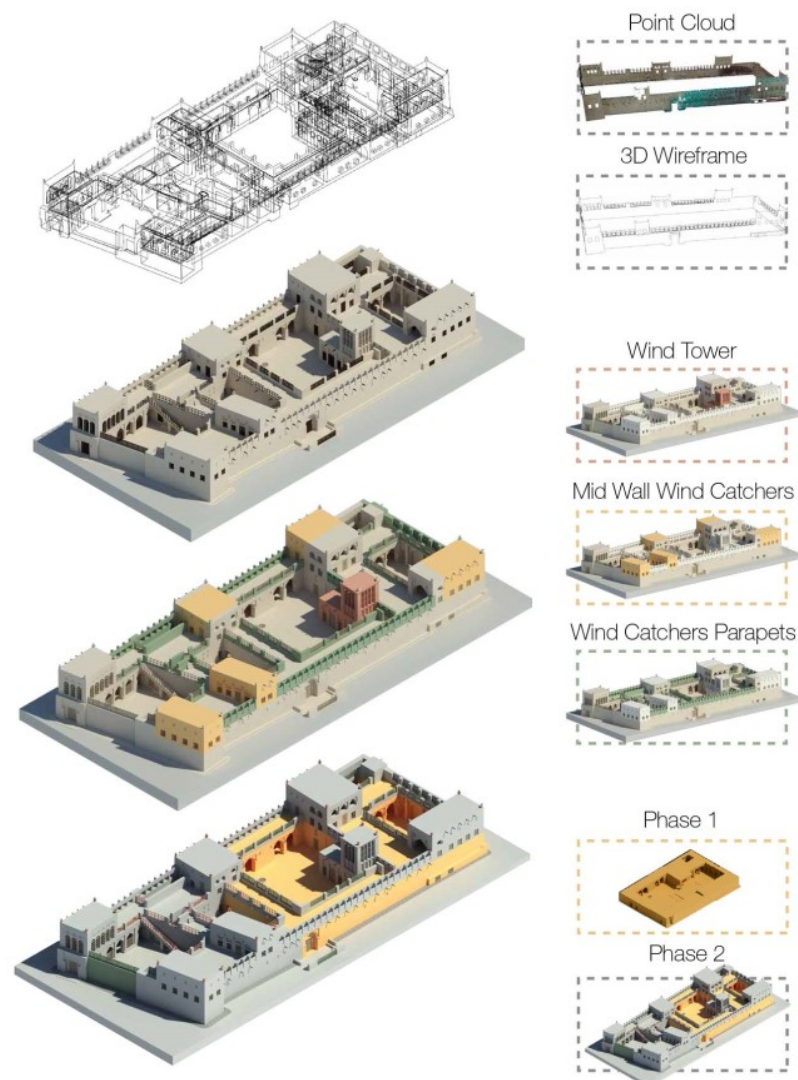

Mid Wall Wind Catchers

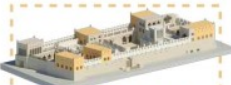

Wind Catchers Parapets
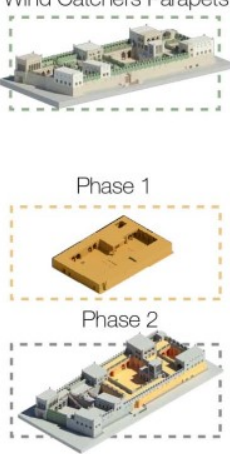

Figure 7. Different HBIM-based analysis realised for the house of Shaykh Isa bin Ali Al-Khalifa.

The "passive" type solutions are quite original; the most ingenious of the systems is called "Baud Geers" (the Persian word means literally "wind-catcher").

In particular, the HBIM climatic analysis of Bahrain (Saeed, 2001), which is based on the Building Bioclimatic Chart, indicated that if a building is designed with the bioclimatic design strategies, they could be utilized for the $34 \%$ of the time of the year. These fascinating devices have been analyzed through Computational Fluid Dynamics (CFD) simulation since the information in a BIM model can be directly extracted for building performance analysis simulation.

The approach to the CFD simulation is based on the definition of the geometry and physical boundary using computer-aided design, the definition of physical modelling and the definition of boundary conditions. It has been possible to generate some graphs showing the way a wind-catcher work. It is mainly based on taking the fresh air into the building and sending the hot and polluted air out.

Therefore, when the ventilation is open on the sided of the wind, there will be a positive pressure to negative pressure.

These graphs have been linked to the HBIM model and the VR project in order to improve the information sharing to different users (BIM experts and non-experts) with different devices (tablet and mobile phone).

Once the information model was ready, there was a need to find a sustainable way of sharing data to all users and professionals involved in the process. 
Examples of Family generated for the BIM model

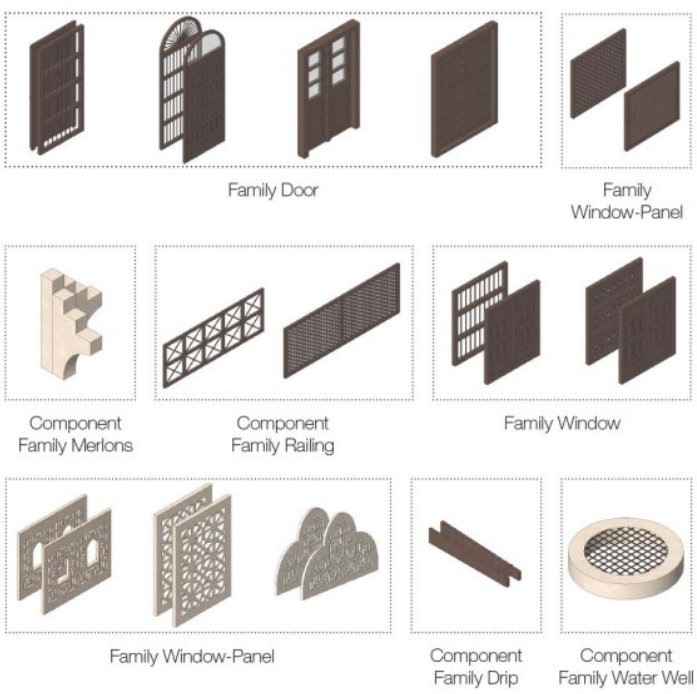

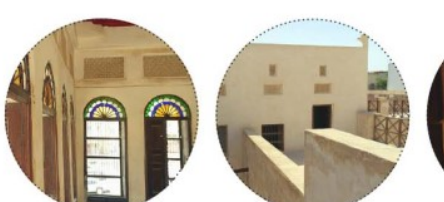
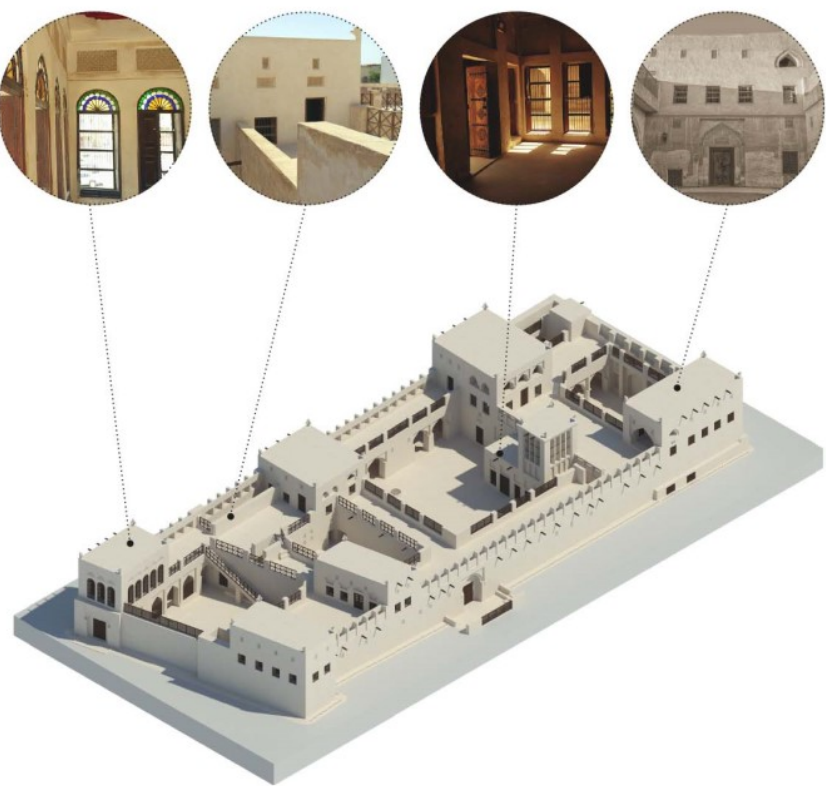

Figure 8. HBIM model, examples of detailed architectural components.

\subsection{HBIM data sharing: open access}

The proposed process was characterised by a method capable of transforming simple 3D surveys into an 'informative' model, which can gather a large amount of information. On the other hand, there is not always the willingness or ability to view information within a BIM project by all users. For this reason, in the last few years, two systems have been chosen to transmit and display information connected to the models (Fig. 9).

HBIM data sharing represents a crucial point. Multi-actors cooperation needs nowadays to be better improved (Della Torre, 2016). O Open access to the HBIM (models and information) can represent valuable support to the multi-actors co-working on the same process. Users that are not familiar with BIM software need to easily access to all the information. It was, therefore, necessary to improve information sharing of different type of data and values such as materials, construction techniques, dating, functions, metric information, volume and areas for the metric computation

The first solution consists of collecting all the data (connected and not connected to the HBIM mode)l and uploading them to a cloud platform able to:

- $\quad$ promote the display of detailed models, drawings and data directly in the browser;

- $\quad$ share data in a simplified way by e-mail or chat, or embed them directly on a website;

- enable model review and shared data in real time through the communication of feedback and comments from all the subjects involved in the project;

- improve the search for data in the digital repository thanks to new filtering functions;

- increase the levels of interoperability thanks to the archiving of any data and any exchange format:

- Reach any data through all types of digital devices (from PC to mobile phone).

The second type of visualisation and data sharing is based on the development of a three-dimensional web interface able to display and connect specific information in the HBIM model for mobile devices (Banfi et al. 2019).

This type of display allowed

- to display the information connected to the model to users who can not manage complex BIM projects. Thanks to the hotspot function (informative windows that can be activated directly from the browser) it is possible to activate info windows connected to the model.

- To avoid using BIM software to view the digital model. This solution has encouraged information sharing via portable devices.

As shown in the next paragraph, this type of display allows you to view the model three-dimensionally through the development of panoramic images from the HBIM model.

The user's interactivity is limited to the web platform where the information related to the model depends on the developer.

For these reasons, this research has developed a VR project able to go beyond the limitations encountered by these two types of web visualisation, favouring an open development over time.

(i)

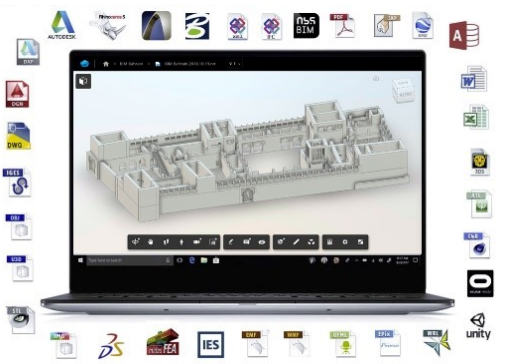

Figure 9. The fourth step: A360 allows information sharing via the cloud. Non-expert users can display HBIM models, data and documentation without having advanced BIM skills. 


\subsection{Interactive Virtual Reality based on HBIM for mobile devices}

Any virtual records of cultural heritage are considered to be a good contribution to the future term. In particular, Visual Storytelling represents a key asset to establishing an emotional connection with the user through captivating, engaging and stimulating narratives. In the last few years, VR allows us to improve our digital worlds for different types of uses. As we well know, technological developments in this specific field of research are aimed at translating the VR experience into the domain of Digital Cultural Heritage.

Different studies show how it is possible to develop innovative experiences for the built heritage and increase the interaction between user and model enriched with information able to tell the story and the richness of the intangible values of the building. In support of these researches, over the years, different types of tools and software have been developed to open the world gaming development to architects and historians who needed to make their knowledge more accessible, favouring a holistic disclosure of models, data and information. It is evident that the process proposed in this study requires different levels of knowledge also from an IT point of view.

For the most part, the skills in modeling and BIM are not sufficient for developing advanced virtual experiences.

Software like Unreal Engine and Unity base their language on the development of codes that increase the user's interaction with the three-dimensional model.

In this context, interaction becomes the key to the improvement of immersive experiences based on digital models, information users and devices. Figure 10 shows the main devices used for user interaction with VR projects.

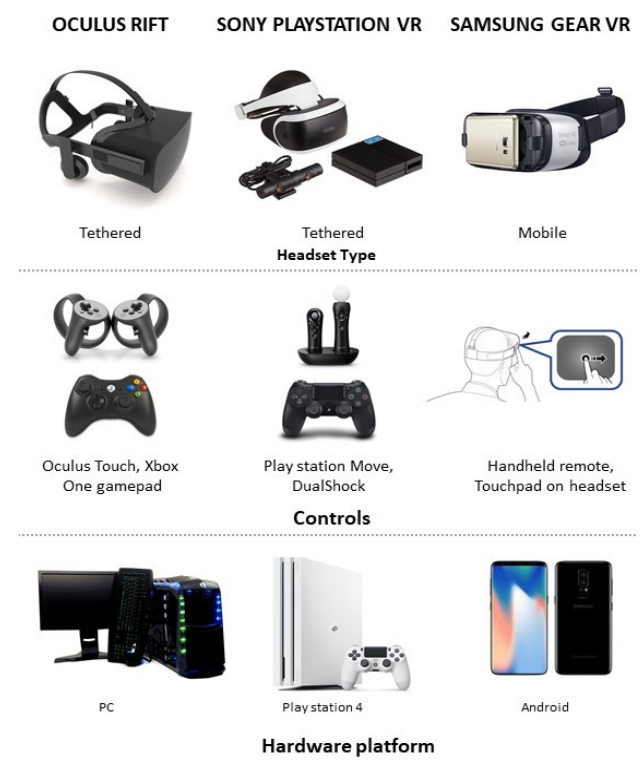

Figure 10. The most commonly used VR devices and software for the development of immersive VR projects.

Different software for the development of VR environments was tested to increase the transmissibility of information and user interaction with an HBIM model. Unreal Engine 4 application proved to be the most suitable.

Thanks to its open language, it was possible to transfer the HBIM model into the software architecture. In particular, this factor has proved to be decisive for the development of a VR project through the Visual Scripting Blueprints.
Unreal Engine, four gameplay scripting system, is based on a structured interface around nodes and allows you to create elements of gameplay directly within the Unreal Editor. It is an extremely powerful, easy-to-use system that deploys the maximum potential in terms of gaming options for video game designers without resorting to computer programming.

This way of developing has turned out to be the operative key for the implementation of complex, immersive VR environments by architects and BIM experts not able to develop IT applications based on computer codes.

Figure 12 shows how Blueprints allow users to set the rules of VR projects, to change the conditions of gameplay, to create different variations of characters, to associate them with materials and information, to change the position of the cameras and their perspective during gameplay, and to manage the $3 \mathrm{D}$ objects for the scenario.

Therefore, Blueprint is an integrated system in Unreal Engine 4 that allows you to visually create game scripts by connecting nodes, events and functions. The Blueprint types are Level Blueprints, Blueprint Classes, Blueprint Macros and Blueprint Interfaces. The great number of Blueprints developed for the research case study shows how the object-information-user interaction can take different forms, from the model to the visual scripting. In this context, the transmissibility of information and the modeling techniques based on GOG 9 and GOG 10 were a determent for the development of a virtual environment based on a Scan-to-BIM model with high levels of detail and information (Fig. 11).
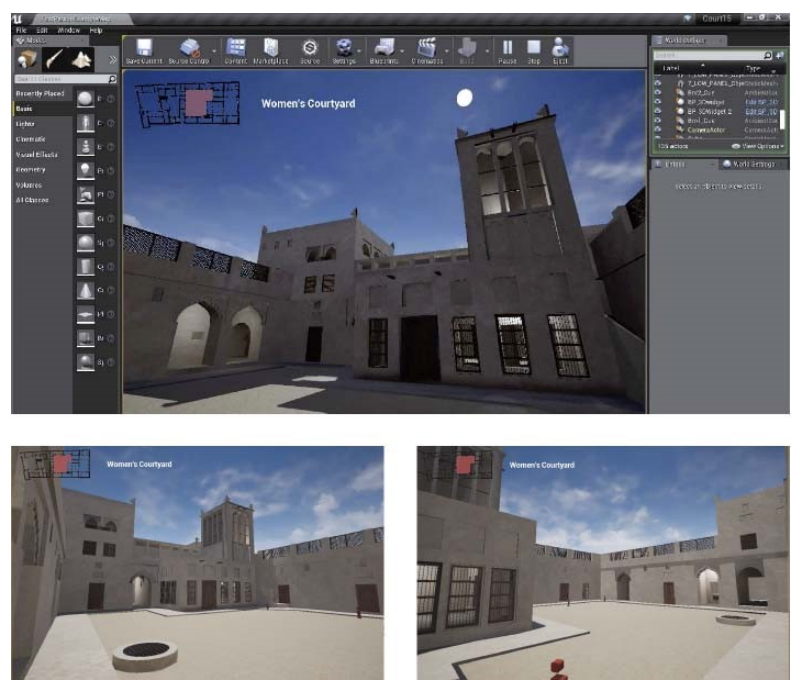

Figure 11. The EU4 VR project based on a detailed HBIM.

The VR experience has been developed for the Smartphone fruition without the headset. It can be called lightweight VR. It offers practical, real-world advantages. Users hold the phone up and away from their faces, and they see a window into another world. The phone is on the touch screen or just move the phone left, right, up or down. In this way, virtual reality could be accessible for people with limited resources. It is often thought of virtual reality as a fancy, expensive, tricky way of capturing someone's attention. Headsets or goggles offer a great immersive experience but they are too much attention outside 
the home, and people are not eager to wear computers on their heads. Smartphone viewing loses the ability to tune out the surroundings. The users will not get the full immersive experience with lightweight VR, but they will get instead is a convenience. Lightweight VR can tell a lot more about a place or even a simple photo. Immersive VR tends to generate heat and use. However, as opposed to gaming, lightweight VR is intended for casual use. It is a potential for a kind of VR usable every day, without the unhandy headsets.

In preparing HBIM information for VR applications has been necessary to simplify the data, specifically the meshes. It is important to reduce the polygon count of the scene to a value compatible with the VR environment. Complex geometries could be affected by errors and superfluous information. For this reason, the first step is to modify and simplify the polysurfaces appropriately. They consist of removing occluded surfaces, repairing NURBS polysurfaces and checking normal to be in the proper orientation. It is important to simplify meshes as much as possible and adding detail via textures, and potentially parallax mapping. Normal Mapping, in $3 \mathrm{D}$ art, is a technique that is used to fake the lighting of bumps and dents from the high polygon object. Normal Maps are based on existing details on a modeled mesh; these details are then projected on a lower polygon mesh; in order to have meshes with a lower polygon count that still keep a good amount of visual detail. Normal maps have been used to reduce the polygon count of the scene. They are based on existing details on a modeled mesh; these details are then projected on a lower polygon mesh; in order to have meshed with a lower polygon. This technique has been used to realise all the decorative gypsum panels by reducing the Polygon Count of almost 50000 triangular polygons.
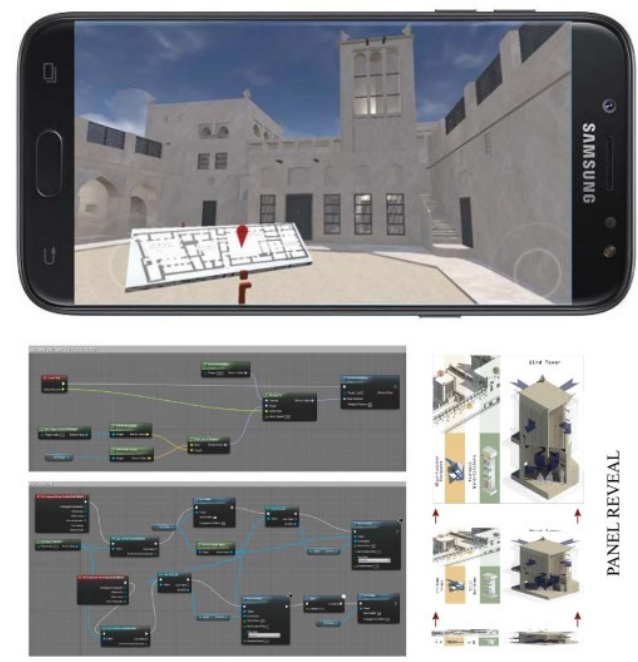

Figure 12. VR project for mobile phones (i) based on EU4 blueprints (ii).

On the other hand, thanks to NURBS modeling it is possible to manage the LOD of detail of each mesh, reducing the conversion of the model for VR projects. A map of the building with the location of the user has been placed in the middle of the courtyard (Fig.12). The first person character can see its position in space, walk over and pick it up. 3D widgets that open and close reveal description and drawings about the architectural and bioclimatic devices. One of the panels reveals a historic photo of the wind tower in order to observe the architectural changes of the building (Fig.13). (i)

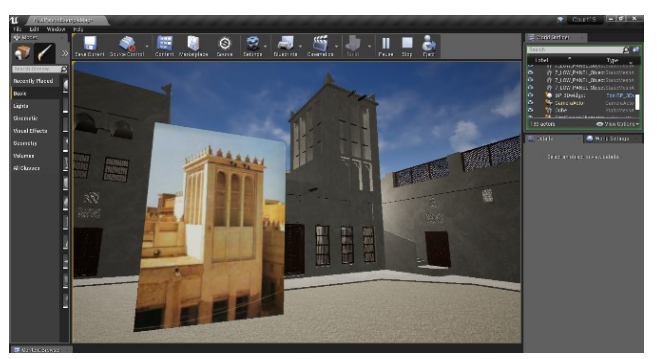

(ii)

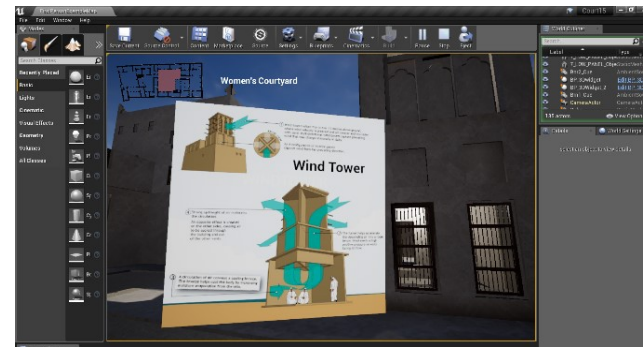

Figure 13. The VR project and its implemented functions. The historic photo of the wind tower in order to observe the architectural changes of the building (i) and 3D widgets that open and close reveal description and drawings about the architectural and bioclimatic devices (ii).

Virtual Reality is a fascinating way to travel; it has the power to transport people to new places. It helps users empathise with situations in other parts of the world. It can lead to wonder and ask questions, especially in this accessible way. Finally, they can contribute to awareness rising of the richness of the constructive elements of the House.

The Virtual Tour of Women's Courtyard provides the opportunity to:

- $\quad$ gain generic information about the heritage building

- develop knowledge about the functioning of the bioclimatic devices

- observe the major changes over time due to the restorations

- $\quad$ appreciate the realistic atmosphere of the environment (structures, details and materials)

\section{RESULT AND CONCLUSIONS}

This paper describes holistic approaches that allow experts to understand the different contributions of the study. This paper, furthermore, contributes to the focus on different ways to make BIM technologies available to experts (by making them able to do their specific analysis) and not-experts (tourist-oriented purposes), increasing information-sharing from 3D survey to immersive VR solutions for our built heritage. The HBIM project of the house has made it possible to highlight a useful research line in order to investigate geometric entities and to associate specific information such as the conservation plan, calculations, and historical phases. This research shows how the use of technological devices help to establish an appropriate conservation process and provide dynamic ways to visual storytelling. 3D Survey, generative modeling, HBIM and a virtual experience inside the building are a powerful instrument 
for dissemination, that collects the deep cultural knowledge of the inhabitants learned over generations that, unfortunately, today they are gradually being forgotten and abandoned.

\section{ACKNOWLEDGEMENTS}

The projects have been carried out in the framework of the "Documentation od Built Heritage at Muharraq for Pearls Path Project" funded by the Bahrain Authority for Culture and Antiquities (BACA). The authors wish to acknowledge and thank the support of BACA's endeavour for the conservation of Bahrain's heritage and for this outstanding opportunity to contribute to this great project. It has been a unique opportunity to collaborate along with other BACA experts in training aimed at improving documentation skills for conservation and preventive maintenance of built heritage. Besides, we wish to thank the support of Noura Al Sayeh, Ahmed Aljishi, Fatima Mahammed Rafiq, Miray Hasaltun Wosisnki, Michal Wosinski and Alaa El Habashi. We also should acknowledge the enthusiasm and participation of the attendees from BACA: Ahmed A.Nabi, Zakariya Abbas, Marwa Reyadh Alarayedh, Nailah Al Khalifa, Dalia Yusuf Abdulhameed, Fatma Yateem, and Fatma A.Nabi, which made this project successful.

\section{REFERENCES}

Banfi, F. (2017). BIM orientation: grades of generation and information for the different type of analysis and management process.

Banfi, F., Chow, L., Ortiz, M. R., Ouimet, C., \& Fai, S. (2018). Building Information Modeling for Cultural Heritage: The Management of Generative Process for Complex Historical Buildings. In Digital Cultural Heritage (pp. 119-130). Springer, Cham.

Banfi, F., Previtali, M., Stanga, C., \& Brumana, R. (2019). A LayeredWeb Interface Based on Hbim and $360^{\circ}$ Panoramas for Historical, Material and Geometric Analysis. ISPRS-International Archives of the Photogrammetry, Remote Sensing and Spatial Information Sciences, $422,73-80$.

Brumana, R., Della Torre, S., Previtali, M., Barazzetti, L., Cantini, L., Oreni, D., \& Banfi, F. (2018). Generative HBIM modelling to embody complexity (LOD, LOG, LOA, LOI): surveying, preservation, site intervention - the Basilica di Collemaggio (L'Aquila). Applied Geomatics, 10(4), 545-567.

Brumana, R., Condoleo, P., Grimoldi, A., Banfi, F., Landi, A. G., \& Previtali, M. (2018). HR LOD based HBIM to detect influences on geometry and shape by stereotomic construction techniques of brick vaults. Applied Geomatics, 10(4), 529-543.

Barazzetti, L., Mezzino, D., \& Quintero, M. S. (2017). Digital workflow for the conservation of Bahrain built heritage: The sheik ISA bin Ali house. INTERNATIONAL ARCHIVES OF THE PHOTOGRAMMETRY, REMOTE SENSING AND SPATIAL INFORMATION SCIENCES, 42(2W5), 65-70.

Barazzetti, L., Banfi, F., Brumana, R., Gusmeroli, G., Previtali, M., \& Schiantarelli, G. (2015). Cloud-to-BIM-to-FEM: Structural simulation with accurate historic BIM from laser scans. Simulation Modelling Practice and Theory, 57, 71-87.

Della Torre, S., (2016). Perspectives on Historic BIM Developments in Italy,AECbytes

(http://www.aecbytes.com/viewpoint/2016/issue_82.html).

Ioannides, M., Magnenat-Thalmann, N., \& Papagiannakis, G. (Eds.). (2017). Mixed Reality and Gamification for Cultural Heritage (pp. 161199). Switzerland: Springer.
Martinelli, P., Galli, A., Barazzetti, L., Colombo, M., Felicetti, R., Previtali, M., ... \& di Prisco, M. (2018). Bearing capacity assessment of a 14th-century arch bridge in Lecco (Italy). International Journal of Architectural Heritage, 12(2), 237-256.

Oreni, D., Brumana, R., Georgopoulos, A., Cuca B., (2013). HBIM for conservation and management of built heritage: towards a library of vaults and wooden beam floors. In: Grussenmeyer, P. (Ed.), ISPRS Annals of Photogrammetry, Remote Sensing and Spatial Information Sciences, vol. II-5/W1. Copernicus Publications. pp. 215-221.

Santana Quintero, M., Cesaro, G., Ishakat, F., Vandesande, A., Vileikis, O., Vadafari, A., ... \& Fakhoury, L. (2012). Protecting Unesco World Heritage PROPERTIES'S Integrity: the Role of Recording and Documentation in Risk Management for PETRA. ISPRS-International Archives of the Photogrammetry, Remote Sensing and Spatial Information Sciences, 121-126.

Stanga, C., Spinelli, C., Brumana, R., Oreni, D., Valente, R., \& Banfi, F. (2017). A nd virtual notebook about the basilica of S. Ambrogio in Milan: information modeling for the communication of historical phases subtraction process.

Trani, M. L., Cassano, M., Todaro, D., \& Bossi, B. (2015). BIM level of detail for construction site design. Procedia Engineering, 123, 581-589.

Saeed, S. A. R. (2001). Energy savings using a bioclimatic architecture with special reference to Bahrain. Architectural Science Review, vol. 44, no. 3, (pp. 277-283).

Nassar, T., Hassan, Y., Abdulnabi, A., Abulfath, S. Shaikh (2017). Isa Bin Ali House General Conservation Directives.

Yarwood, J. (2005). Al-Muharraq: Architectural Heritage of a Bahraini City. Manama: Miracle Graphics. 